\title{
Diboson and top quark pair production cross section measurements at 5.02 TeV in CMS
}

\section{Andrea Trapote Fernández* on behalf of the CMS Collaboration}

Universidad de Oviedo, Instituto Universitario de Ciencias y Tecnologías Espaciales de Asturias (ICTEA) Oviedo, Spain

E-mail: andrea.trapote.fernandez@cern.ch

We explore diboson and top quark pair productions in proton-proton collisions at a new centreof-mass energy, $5 \mathrm{TeV}$, using $304 \mathrm{pb}^{-1}$ of data collected in 2017 by the CMS experiment at the LHC. The diboson, WW, WZ, and ZZ, cross sections are measured analyzing events with two, three or four charged leptons in the final state. The measured cross sections are compared with NNLO predictions and across other experiments. The top quark pair production measurement is performed using events with one electron and one muon of opposite sign, and at least two jets. To reduce the statistical uncertainty, a combination with the result in the $1+$ jets channel, based on $27.4 \mathrm{pb}^{-1}$ of data collected in 2015 at the same center-of-mass energy, is then performed.

The European Physical Society Conference on High Energy Physics (EPS-HEP2021) 26-30 July 2021

Online conference, jointly organized by Universität Hamburg and the research center DESY

\footnotetext{
* Speaker
} 


\section{Contents}

1 Introduction 2

2 Diboson cross section measurement 2

$3 t \bar{t}$ cross section measurement 3

\section{Introduction}

A new energy centre-of-mass energy is explored for the diboson $\left(W^{+} W^{-}, W^{ \pm} Z\right.$ and $\left.Z Z\right)$ [1] and top quark pair production $(t \bar{t})$ [2]. These processes are important. They are tests of the Standard Model (SM) of particle physics and irreducible backgrounds in other SM measurements and beyond the SM searches.

In this publication, measurements of the diboson and $t \bar{t}$ production cross sections in protonproton collisions at a center-of-mass energy of $5.02 \mathrm{TeV}$ at the CERN LHC, corresponding to an integrated luminosity of $304 \mathrm{pb}^{-1}$, are presented. These analyses are performed using a data set recorded with the CMS experiment [3] in 2017 and it is characterized by a relatively low number of additional interactions per bunch crossing as compared to the measurements performed at higher center-of-mass energies.

\section{Diboson cross section measurement}

In the case of the diboson production measurement, the object selection relies on precise lepton identification which is achieved using a MVA discriminant trained [4] to separate between prompt and nonprompt lepton sources.

Several signal regions (SR) are defined to properly distinguish from $W^{+} W^{-}, W^{ \pm} Z$ and $Z Z$ contributions using variables such as the multiplicity and flavour of the leptons involved in the final state, the transverse momentum of the leptons, the minimum amount of missing energy, the number of jets in the final state and consistency between the invariant mass of a lepton pair, originated after a $\mathrm{Z}$ boson decay, and the mass of the $\mathrm{Z}$ boson.

Most background contributions, including photon conversions, charge mismeasurement, and those processes yielding prompt leptons in the final state, such as $t \bar{t}$, single top, Drell-Yan(DY), and diboson production, are estimated from simulation. Backgrounds involving one or more nonprompt leptons are estimated from simulation aided by control samples in data in those categories with two leptons in the final state and exclusively from simulation otherwise.

The number of events in the SRs are measured in a counting experiments in regions with high signal purity. The total cross section is measured as:

$$
\sigma=\frac{N_{\text {signal }}^{\mathrm{SR}}}{\mathcal{B R}(V \rightarrow X X) \mathcal{B R}(V \rightarrow X X) \epsilon \mathcal{L}},
$$


where $\mathcal{L}$ is the total integrated luminosity, $\epsilon$ the efficiency of the lepton reconstruction and the additional phase space requirements, and $N_{\text {signal }}^{S R}$ is the number of obtained signal events, estimated for each SR by performing a maximum likelihood fit to the yields with a single free-floating parameter that corresponds to the normalization of the signal process.

The measured cross sections for the WW, WZ, and ZZ productions, shown in 1, are [1]: $\sigma_{W W}=$ $36.5_{-5.1}^{+5.5}(\text { stat })_{-2.5}^{+2.6}($ syst $) \mathrm{pb}, \sigma_{W Z}=6.4_{-2.1}^{+2.4}(\text { stat })_{-0.3}^{+0.5}($ syst $) \mathrm{pb}$ and $\sigma_{Z Z}=5.3_{-2.0}^{+2.5}(\text { stat })_{-0.4}^{+0.5}($ syst $)$ $\mathrm{pb}$. These values are consistent with MATRIX predictions at next-to-next-to-leading-order (NNLO) QCD times next-to-leading order electroweak cross sections (NNLO QCD×NLO EWK).

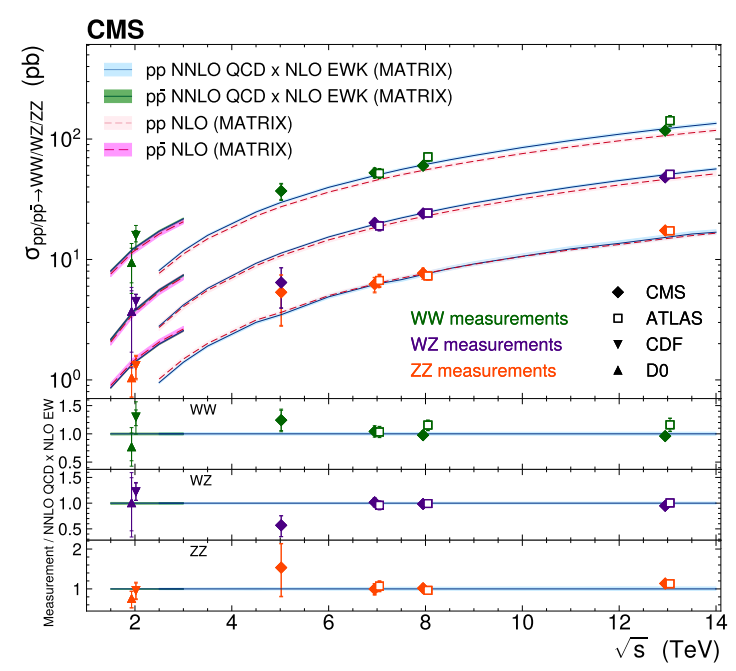

Figure 1: Results obtained in this analysis and other diboson production cross section measure-ments at different center-of-mass energies for the CMS, ATLAS, CDF, and D0 Collaborations are presented, and compared with the NNLO QCD $\times$ NLO EW and NLO predictions from MATRIX [5]. The vertical error bars represent the uncertainty in the measured cross section. [1]

\section{3. $t \bar{t}$ cross section measurement}

For top quark pair production cross section measurement, events with one electron and one muon with opposite charge are selected. In addition, events are required to have at least two jets and a dilepton invariant mass above $20 \mathrm{GeV}$ to reduce DY and other backgrounds from low mass resonances and from photon conversions.

After the event selection, background events arise mainly from tW, DY, and VV production in which at least two prompt leptons emerge from the $\mathrm{Z}$ or $\mathrm{W}$ boson decays. The tW and VV contributions are estimated from simulation and the DY events are estimated from data.

The $t \bar{t}$ production cross section is extracted by performing a counting experiment:

$$
\sigma=\frac{N-N_{\mathrm{bkg}}}{\epsilon \mathcal{A B \mathcal { L }}}
$$


where $N$ is the number of observed events, $N_{\mathrm{bkg}}$ is the number of estimated background events, $\epsilon$ is the event selection efficiency, $\mathcal{A}$ is the total acceptance, $\mathcal{B}$ is the branching fraction of $\mathrm{W}$ boson pair $t \bar{t} \rightarrow e^{ \pm} \mu^{\mp}$ of $3.194 \%$ [6], and $\mathcal{L}$ is the integrated luminosity.

The measured inclusive cross section for a top quark mass of $172.5 \mathrm{GeV}$ is: $\sigma_{t \bar{t}}=60.3 \pm$ 5.0 (stat $) \pm 2.8($ syst $) \pm 0.9$ (lumi) $\mathrm{pb}=60.3 \pm 5.5$ (tot $) \mathrm{pb}$. This result is combined with that obtained in the $\ell+$ jets decay channel [7], corresponding to a luminosity of $27.4 \mathrm{pb}^{-1}$, to reduce the statistical limitation: $\sigma_{t \bar{t}}=62.6 \pm 4.1($ stat $) \pm 3.0($ syst + lumi $) \mathrm{pb}=62.6 \pm 5.0($ tot $) \mathrm{pb}$. Both results are in agreement with the SM prediction at NNLO in QCD including soft-gluon resummation at NNLL, as shown in Figure 2.

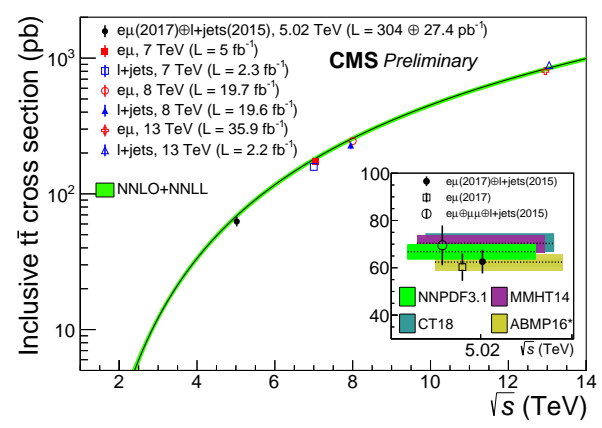

Figure 2: Summary of CMS measurements of $\sigma_{t \bar{t}}$ in pp collisions at different center-of-mass energies, compared to the NNLO+NNLL prediction. The vertical bars and bands represent the total uncertainties in the data and in the predictions respectively. [2]

\section{References}

[1] CMS Collaboration, Measurements of the electroweak diboson production cross sections in proton-proton collisions at 5.02 TeV using leptonic decays, arXiv:2107.01137

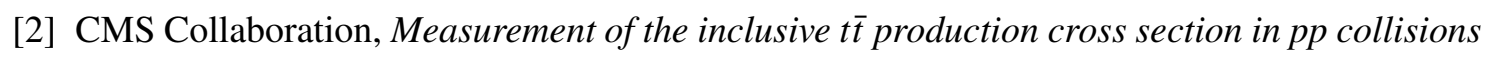
$5.02 \mathrm{TeV}$, CMS-PAS-TOP-20-004

[3] CMS Collaboration, The CMS experiment at the CERN LHC, JINST 3 (2008) S08004. doi:10.1088/1748-0221/3/08/s08004

[4] CMS Collaboration, Measurement of the Higgs boson production rate in association with top quarks in final states with electrons, muons, and hadronically decaying tau leptons at $13 \mathrm{TeV}$, Eur. Phys. J. C 81 (2021) 378. doi:10.1140/epjc/s10052-021-09014-X

[5] M. Grazzini, S. Kallweit and M. Wiesemann, Fully differential NNLO computations with MATRIX, Eur. Phys. J. C 78 (2018) 7. doi:10.1140/epjc/s10052-018-5771-7

[6] Particle Data Group, Review of particle physics, Phys. Rev. D. 098 (2018) 030001. doi:10.1103/PhysRevD.98.030001

[7] CMS Collaboration, Measurement of the inclusive $t \bar{t}$ cross section in $p p$ collisions at $5.02 \mathrm{TeV}$ using final states with at least one charged lepton, JHEP 03 (2018) 115. doi:10.1007/JHEP03(2018)115 